\title{
Cacaoculture agroforestière en Afrique : l'art de concilier production durable et services écologiques
}

\author{
Patrick Jagoret - Stéphane Saj - Aurélie Carimentrand
}

L'agroforesterie, qui associe des arbres aux cacaoyers, apparaît comme une option crédible pour renouveler la cacaoculture en Afrique. La culture pure de cacaoyers, dominante, y est en effet à bout de souffle. Il est donc urgent d'identifier des solutions techniques pour stabiliser les zones cacaoyères, réduire la tension sur les forêts et s'adapter à l'évolution du climat. Un équilibre peut ètre trouvé entre les cacaoyers et un panachage $d$ 'arbres choisis par les agriculteurs pour leurs usages variés, tout en maintenant un rendement en cacao convenable sur le long terme. Cet équilibre assure aussi des services écologiques comme le stockage du carbone et la protection phytosanitaire. Des travaux récents menés au Cameroun montrent que le pilotage des cacaoyères agroforestières peut s'appuyer sur un indicateur simple, la mesure de la surface terrière des cacaoyers et celle des arbres associés. Cet indicateur pourrait être adopté pour la certification du cacao durable, tandis que la convergence constatée entre savoirs locaux et résultats scientifiques pourrait aider à co-construire les conseils techniques.
Ces 60 dernières années, la cacaoculture s'est fortement développée en Afrique où le verger cacaoyer a doublé, passant de 3,3 à 6,5 millions d'hectares. Avec ses trois millions de tonnes de cacao par an (865 000 tonnes en 1961), le continent africain est devenu le leader mondial de la production. Les principaux pays producteurs sont la Côte d'Ivoire et le Ghana, qui couvrent à eux seuls $70 \%$ de l'offre mondiale.

Si le rendement moyen du verger cacaoyer africain a presque doublé lui aussi, passant de 254 à 484 kilogrammes par hectare entre 1961 et 1996, il n'a pratiquement pas évolué depuis. Force est donc de constater que l'accroissement de la production mondiale a toujours été indissociable de l'extension des surfaces cultivées, le coût de la réhabilitation d'une cacaoyère dégradée étant supérieur à celui de l'installation d'une nouvelle cacaoyère après défriche forestière.

En Côte d'Ivoire et au Ghana, l'expansion du verger cacaoyer a ainsi contribué à une déforestation massive avec, en corollaire, la perte de nombreux services écologiques - conservation de la biodiversité, stockage du carbone, maintien de la fertilité des sols. La culture pure de cacaoyers [" monoculture ", sans arbres associés ou très peu] y est historiquement dominante, mais elle est actuellement dans l'impasse car peu durable. En effet, les vergers anciens, faute d'apports adéquats d'intrants, ne produisent quasiment plus après 20-30 ans et les techniques de réhabilitation proposées (replantation, taille) y sont rarement appliquées. En outre, les espaces forestiers disponibles pour installer de nouveaux vergers se font rares, amenant de nombreux agriculteurs à occuper les forêts classées. À cela s'ajoute le changement climatique, qui engendrera en Afrique de l'Ouest une limitation des zones propices à la cacaoculture. Face à la menace de voir leurs dernières forêts disparaître, la Côte d'Ivoire et le Ghana, rejoints par les principales entreprises de l'industrie du cacao, se sont donc engagés à la Conférence de Bonn sur le climat en 2017 (COP23) à les protéger et à promouvoir l'agroforesterie.

Aujourd'hui, il est urgent d'identifier des solutions techniques solides qui permettront à cette région de faire face à deux grands défis: d'une part, stabiliser les zones cacaoyères existantes et réduire la tension croissante sur les forêts résiduelles et, d'autre part, s'adapter à l'évolution du climat.

De même, des alternatives à la monoculture doivent être réfléchies dans les pays possédant encore de vastes forêts, notamment en Afrique centrale. Ces pays pourraient en effet légitimement diversifier leur économie en développant la cacaoculture, complétant ainsi les capacités de production de l'Afrique de l'Ouest dont le plafond pourrait être atteint.

\section{L'agroforesterie, option crédible pour}

\section{le renouveau de la cacaoculture africaine}

Depuis les années 1960, l'agroforesterie, c'est-à-dire le fait d'associer des arbres fruitiers et forestiers aux cacaoyers, est déconseillée aux agriculteurs. Encore aujourd'hui, il est reproché à cette pratique traditionnelle des rendements 
plus faibles qu'en monoculture, alors qu'elle perdure dans de nombreux pays producteurs. Les arbres, associés en plus ou moins grand nombre, induiraient une compétition pour la lumière, l'eau et les éléments minéraux, au détriment des cacaoyers.

Si ces arguments sont pertinents, des travaux récents menés au Cameroun montrent que la présence d'arbres dans les cacaoyères n'empêche pas des rendements corrects. Dans ce pays, le verger est surtout constitué de cacaoyères agroforestières. Le rendement, estimé à partir de comptages des cabosses, est en moyenne de 740 kilogrammes de cacao marchand par hectare dans des parcelles où se côtoient en moyenne 1500 cacaoyers - densité similaire à celle souvent observée en monoculture - et 190 arbres fruitiers et forestiers. Il peut même atteindre une tonne alors qu'aucun engrais chimique n'a jamais été apporté. De plus, en fonction de la complexité de ces cacaoyères (nombre et types d'arbres associés), leur capacité moyenne de stockage de carbone peut atteindre 75 tonnes par hectare, voire plus, ce qui représente $50 \%$ de celle des forêts secondaires locales, où elles ont souvent été installées. La capacité de stockage de carbone des monocultures de cacaoyers est de l'ordre de 10 tonnes.

Dans certaines parcelles, ce compromis entre production de cacao et stockage de carbone va de pair avec un autre service écologique crucial pour les agriculteurs, la régulation de deux bioagresseurs majeurs : la pourriture brune des cabosses, due à un champignon, et les mirides, insectes piqueurs-suceurs dont les dégâts répétés entraînent la mort progressive des cacaoyers. Les agriculteurs modulent l'ombrage procuré par les arbres associés pour limiter à la fois le développement du champignon (favorisé par un ombrage dense) et les mirides (qui pullulent lorsque l'ombrage est absent ou léger). Ils parviennent ainsi à réduire l'apport de pesticides et à économiser jusqu'à $70 \%$ du budget de protection phytosanitaire d'une parcelle conduite de façon intensive.

\section{Un indicateur opérationnel pour le pilotage d'une cacaoyère agroforestière : la surface terrière}

L'observation des cacaoyères agroforestières au Cameroun a mis en évidence que la surface terrière des cacaoyers, reflet des compétitions entre les cacaoyers et les arbres associés, est un bon indicateur pour évaluer les niveaux de compromis entre la production de cacao et les services écologiques. II s'agit de mesurer la circonférence du tronc à une hauteur donnée, puis de calculer la surface de la section transversale, ou " surface terrière ». Classiquement utilisée par les forestiers, cette mesure peut être aisément applicable par les techniciens, voire les agriculteurs eux-mêmes.

L'indicateur global qui nous intéresse ici est la surface terrière relative des cacaoyers (voir encadré p. 3), c'est-à-dire le ratio entre la somme des surfaces terrières de tous les cacaoyers et celle des surfaces terrières de tous les arbres présents dans la parcelle (cacaoyers + arbres associés). Au Cameroun, la valeur de cet indicateur est en moyenne de $40 \%$ dans les cacaoyères agroforestières adultes qui produisent une tonne de cacao marchand par hectare. Elle est de $36 \%$ dans les cacaoyères qui présentent les meilleurs compromis entre rendement en cacao, stockage de carbone et régulation des bioagresseurs.

Cet indicateur permet aussi d'évaluer un autre compromis qui concilie bon niveau de production de cacao et longévité de la cacaoyère. Au Cameroun, les surfaces terrières relatives des cacaoyers comprises entre 40 et $55 \%$ permettent à la fois des rendements jusqu'à une tonne de cacao par hectare et le maintien des cacaoyères après 40 ans, seuil au-delà duquel il est généralement admis qu'elles doivent être réhabilitées.
Les agriculteurs camerounais parviennent à cet équilibre en réduisant la densité des arbres associés au cours du temps, pour n'en conserver que 120 à 140 par hectare dans les cacaoyères les plus âgées. Ils stabilisent ainsi la surface terrière de ces arbres autour de $16 \mathrm{~m}^{2}$ par hectare, au bénéfice des cacaoyers dont la surface terrière augmente, passant de 2,4 $\mathrm{m}^{2}$ par hectare dans les jeunes parcelles à $9 \mathrm{~m}^{2}$ dans les plus anciennes. Ces pratiques de gestion des arbres associés sur le long terme vont de pair, en particulier dans les parcelles sénescentes, avec la réhabilitation progressive des cacaoyers, qui consiste à remplacer les individus morts et à tailler les vieux cacaoyers pour renouveler leurs troncs (taille de " recépage "). Dans les cacaoyères dégradées, la présence d'arbres permet aux agriculteurs de s'affranchir de deux préalables à toute action de réhabilitation : la restauration d'un ombrage, celui-ci étant déjà présent, et celle de la fertilité du sol, maintenue par la décomposition des débris végétaux.

Le panachage d'espèces d'arbres réalisé par les agriculteurs dépend de leurs usages (fruits autoconsommés ou vendus, bois d'œuvre, écorces médicinales, etc.) et des services qu'ils peuvent rendre (maintien de la fertilité du sol, ombrage, etc.]. Les agriculteurs distinguent ces espèces selon leurs effets positifs ou négatifs sur les cacaoyers. Ils font volontairement des compromis entre la production de cacao et les autres usages et services en fonction de leur stratégie de production et de leur capacité économique. Même si la variabilité des rendements observée au Cameroun suggère que le mélange d'espèces et leur pilotage restent à mieux maîtriser pour nombre d'agriculteurs, les connaissances empiriques sont régulièrement cohérentes avec les connaissances scientifiques. Elles pourraient légitimement servir de base au dialogue entre agriculteurs et chercheurs, la convergence entre savoirs locaux et résultats scientifiques permettant de co-construire un conseil technique adapté aux attentes des agriculteurs.

En conclusion, la surface terrière relative des cacaoyers apparaît comme un indicateur clé de pilotage d'une cacaoyère agroforestière pour gérer ses performances agronomiques, sa longévité et les compromis entre le rendement en cacao et différents services écologiques. Ce type d'analyse, obtenu grâce à une mesure simple sur les cacaoyers et les arbres associés, peut être facilement réalisé dans d'autres zones de cacaoculture ou d'autres situations environnementales. Il serait alors possible de préciser localement les valeurs de cet indicateur de surface terrière et celles des densités des arbres associés. Cela amènerait à mieux estimer les compromis existants et à défınir les niveaux optimaux atteignables localement (rendement en cacao, stockage de carbone, etc.). Ce calibrage étant établi, il constituerait une base pour réfléchir avec les agriculteurs aux leviers techniques leur permettant d'atteindre les compromis souhaités.

\section{La nécessaire évolution des critères de certification}

Depuis la fin des années 1990, les standards volontaires de durabilité et de commerce équitable de la filière cacao (Rainforest Alliance, UTZ, Fairtrade, etc.) visent à améliorer les performances de productivité et de durabilité des cacaoyères. Dotés de systèmes de certification, ces standards sont censés apporter la garantie qu'un ensemble de critères de durabilité est respecté au moyen de différentes procédures élaboration et révision des cahiers des charges puis des critères de conformité, protocoles d'audit, formation et autorisation des organismes certificateurs. Les cahiers des charges de ces standards comprennent ainsi un ensemble de critères de gestion agricole, sociaux et environnementaux. Les critères relatifs aux pratiques agroforestières diffèrent toutefois d'un standard à l'autre : il peut s'agir d'un nombre d'arbres adultes présents dans la cacaoyère (12 arbres par hectare par exemple), 
Une surface terrière relative des cacaoyers de l'ordre de 40 à $55 \%$, gage d'un bon compromis entre rendement en cacao et longévité de la cacaoyère

Dans cette étude menée au Cameroun (région du Centre) chaque point de la figure représente une cacaoyère et sa taille est proportionnelle à son rendement en cacao marchand les rendements allant de moins de 50 kilogrammes à plus de deux tonnes par hectare.

\section{Surface terrière 100 relative des
cacaoyers [\%]}
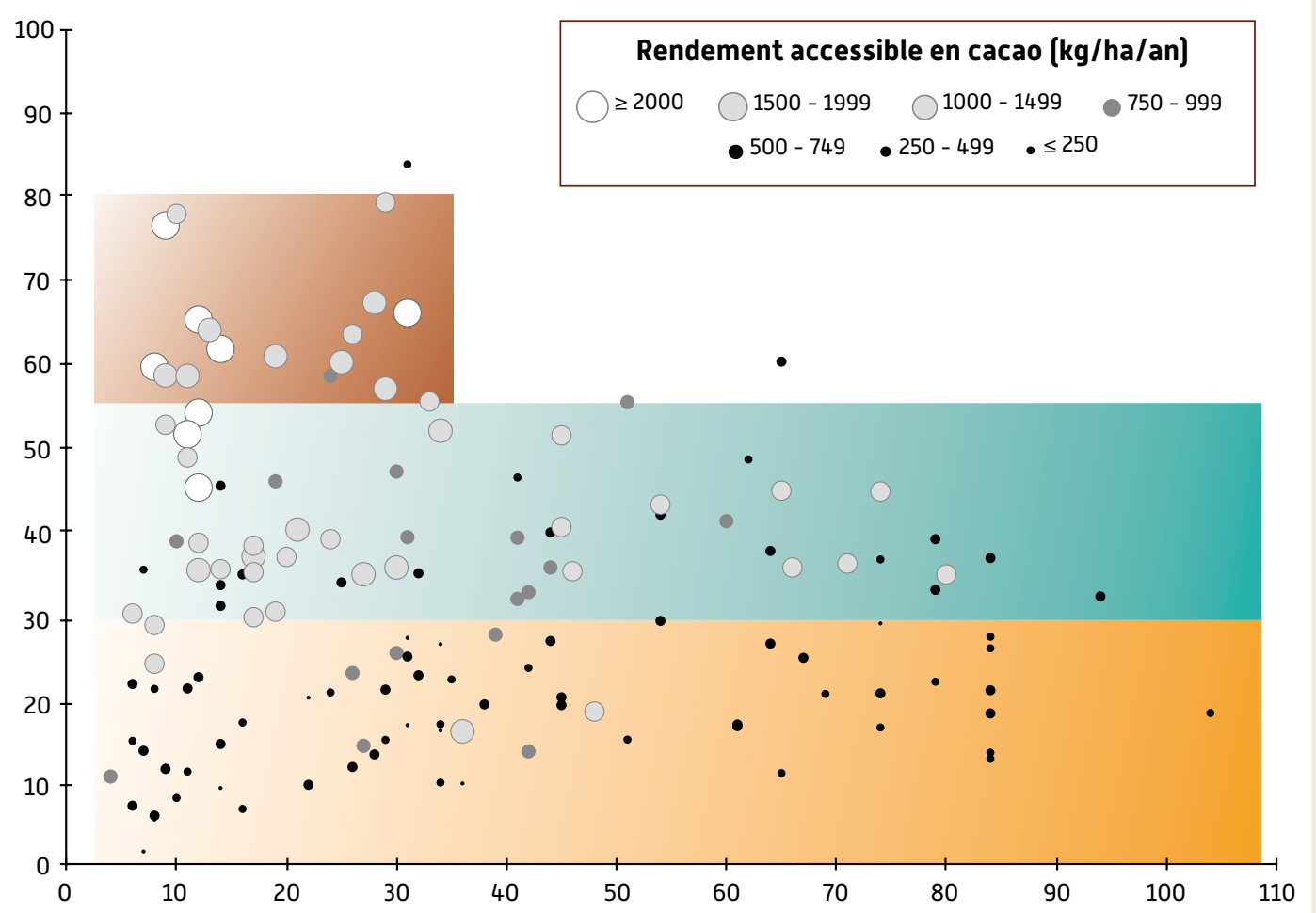

Source : d'après Saj et al., 2017. https://doi.org/10.1016/j.agsy.2017.06.002

Âge de la cacaoyère [années]

Dans la partie centrale de la figure [bleu], les cacaoyères agroforestières ont des caractéristiques optimales. Les rendements sont proches ou supérieurs à une tonne de cacao par hectare, et elles durent bien au-delà de 40 ans. Elles comportent en moyenne 137 arbres associés par hectare. La surface terrière relative des cacaoyers est comprise entre 30 et $55 \%$ - les cacaoyers représentent en moyenne $9,3 \mathrm{~m}^{2}$ et les arbres associés $11,4 \mathrm{~m}^{2}$.

Dans la partie haute de la figure (marron), les cacaoyères ne sont pas durables. Elles sont simples, avec en moyenne 70 arbres associés par hectare. La surface terrière relative des cacaoyers y est supérieure à $55 \%$ - les cacaoyers représentent en moyenne $8,6 \mathrm{~m}^{2}$ et les arbres associés $3,8 \mathrm{~m}^{2}$. Le rendement peut atteindre plus de deux tonnes par hectare, mais ces cacaoyères ne durent pas plus de 30 à 40 ans car il est difficile de les conserver, même en recourant à des apports d'intrants chimiques (fertilisants notamment).

À l'opposé, dans la partie basse de la figure [orange], les cacaoyères sont durables mais produisent peu. Elles sont complexes, avec 176 arbres associés par hectare. La surface terrière relative des cacaoyers y est inférieure à $30 \%-$ les cacaoyers représentent $5,1 \mathrm{~m}^{2}$ et les arbres associés $24,4 \mathrm{~m}^{2}$. Le rendement est de moins de 50 kilogrammes à 750 kilogrammes par hectare. d'une couverture aérienne des arbres d'ombrage $30 \%$ par exemple) combinée à un nombre d'espèces associées (cinq espèces par exemple), ou encore d'un couvert de végétation indigène (15\%).

Ces standards ont été largement adoptés depuis les années 2010 et ont permis d'initier une dynamique vertueuse de pratiques agroforestières en cacaoculture. Mais leurs critères présentent de sérieuses limites par rapport à la fourniture de services écologiques et à la longévité de la cacaoyère : le bénéfice à attendre apparaît en effet assez marginal pour le stockage de carbone, la biodiversité, la régulation des bioagresseurs et le maintien de la fertilité des sols.

D'abord, les travaux conduits au Cameroun montrent que le bénéfice attendu des arbres associés - c'est-à-dire des compromis intéressants entre production de cacao, plusieurs services écologiques et longévité de la cacaoyère - suppose d'en conserver une centaine par hectare. C'est beaucoup plus que les recommandations des programmes de certification actuels.
Ensuite, le critère de couverture aérienne des arbres d'ombrage est très difficile à quantifier, ce qui le rend interprétable et en réduit la fiabilité. L'ombrage est en effet un paramètre extrêmement variable et les différentes techniques pour l'estimer présentent de multiples contraintes. Il en est de même pour le couvert de végétation indigène dont la définition peut aussi être sujette à caution.

Enfin, les critères retenus - densité des arbres, nombre d'espèces, ombrage, couverture de végétation, indigène ou non - ne permettent pas d'évaluer réellement les niveaux de compromis entre production de cacao et services écologiques.

C'est pourquoi la surface terrière relative des cacaoyers apparaît comme un critère de conformité dont ces standards pourraient s'emparer pour disposer d'un nouvel outil de pilotage des cacaoyères : indicateur précis, facilement mesurable et surtout adaptable aux conditions locales.

Pour atteindre les objectifs fixés par les pays européens signataires de la déclaration d'Amsterdam (2015) qui se sont 
engagés à ne plus admettre d'importations de cacao non certifié et à développer des stratégies de lutte contre la déforestation importée, il est toutefois nécessaire que l'adoption de cet indicateur dans le panel des critères de conformité pour la certification du cacao durable s'accompagne de mesures de financement pour les agriculteurs. II pourrait s'agir de primes revues à la hausse et mieux distribuées, $d$ 'incitations financières découplées de la productivité comme les paiements pour services environnementaux [PSE], ou d'une fiscalité incitative (système de bonus-malus fiscal pour favoriser le cacao durable par exemple]. Ces leviers économiques sont indispensables pour aider les producteurs de cacao à s'adapter aux standards de production qui leur sont imposés et à adopter des pratiques agroforestières nécessaires au renouveau de la cacaoculture africaine.
Ce Perspective $\mathrm{n}^{\circ} 54$ est issu de travaux de recherche et d'expertise conduits d'une part sur les systèmes agroforestiers cacaoyers par le Cirad et l'Institut de recherche agricole pour le développement (Irad) au Cameroun et, d'autre part, sur la certification du cacao par le Cirad pour I'Agence française de développement [Afd, https://www.afd.fr/fr]

Au Cameroun, les travaux ont été réalisés de 2009 à 2017 dans le dispositif de recherche et d'enseignement en partenariat du Cirad (dP) Systèmes agroforestiers en Afrique centrale - Agroforesterie Cameroun (https://www. cirad.fr/nos-recherches/dispositifs-en-partenariat/liste-des-dispositifs/ agroforesterie-cameroun) via les projets suivants :

$>$ STRADIV System approach for the TRAnsition to bio-DIVersified agroecosystems (https://stradiv.cirad.fr/), 2015-2018, financé par la fondation Agropolis (https://www.agropolis-fondation.fr/)

> SAFSÉ, Recherche de compromis entre productions et services écosystémiques fournis par les systèmes agroforestiers tropicaux (https://safse.cirad.fr/), 2012-2015, financé par le Cirad et l'Institut de recherche pour le développement (Ird, https://www.ird.fr/);

$>$ AFS4FOOD, Améliorer la sécurité alimentaire et le bien-être des ménages ruraux africains à travers une meilleure synergie entre les systèmes agroforestiers et les cultures vivrières (https://afs4food.cirad.fr/], 2012-2015, projet contracté par I'Union Africaine sur un financement de l'Union européenne [EuropAid] ;

$>$ REPARAC, Renforcement des partenariats dans la recherche agronomique au Cameroun, 2005-2008, financé par le ministère français des Affaires étrangères (Pedelahore P., et al., 2009. Résultats et enseignements du projet REPARAC. In Atelier PCP-REPARAC Innover pour améliorer les revenus des exploitations familiales et la production agricole du Grand Sud Cameroun, 24-26 juin 2009, Mbalmayo, Cameroun. Cirad, 21 p. http://agritrop.cirad.fr/550870/]

\section{Quelques mots sur..}

Patrick Jagoret est agronome au Cirad à I'Umr ABSys (Agrosystèmes biodiversifiés, https://umr-absys.cirad.fr/]. Ex-directeur adjoint de I'Umr System (Fonctionnement et conduite des systèmes de culture tropicaux et méditerranéens), il conduit des recherches sur le fonctionnement des cacaoyères agroforestières. II pilote le projet européen DESIRA (Development-Smart Innovation through Research in Agriculture) Cocoa4Future, Durabilité des systèmes de production et dynamiques nouvelles du secteur cacaoyer en Côte d'Ivoire et au Chana, lancé en 2020 (https://europa.eu/capacity4dev/desira/wiki/cocoa4future). patrick.jagoret@cirad.fi

Stéphane Saj est agronome au Cirad à I'Umr ABSys en Guyane à Kourou. Responsable de l'équipe SAFT (Systèmes agroforestiers tropicaux), il travaille sur le fonctionnement et la conception de systèmes agroforestiers, avec les objectifs suivants : augmenter le niveau de vie des agriculteurs et leur accès à la sécurité alimentaire ; aboutir à des systèmes agroforestiers ayant une meilleure résistance aux aléas et changements climatiques. II étudie les compromis entre les services écosystémiques pouvant être fournis par ces systèmes complexes.

stephane.saj@cirad.fr

Aurélie Carimentrand est économiste de la qualité dans l'équipe Equaliter [Équité, qualité, alimentation, territoire] de l'Umr Innovation (Innovation et développement dans l'agriculture et I'alimentation, https://umr-innovation.cirad.fr/]. Basée à Montpellier, elle travaille sur les systèmes de certification de la qualité sociale, environnementale et d'origine des produits alimentaires, sur les innovations socio-économiques et territoriales en lien avec ces systèmes et sur leurs impacts. aurelie.carimentrand@cirad.fr
Ces travaux ont donné lieu à des publications, parmi lesquelles:

Andreotti F., Mao Z., Jagoret P., Speelman E. N., Gary G., Saj S., 2018. Exploring management strategies to enhance the provision of ecosystem services in complex smallholder agroforestry systems. Ecological Indicators 94 (Part 1): 257-265. https://doi.org/10.1016/j.ecolind.2018.06.048

Carimentrand A., 2020. Cacao : état des lieux sur la déforestation et les standards de durabilité. Rapport d'étude du Cirad. Commanditaire : Comité scientifique et technique Forêts de l'Agence française de développement (Afd]. Montpellier, Cirad, 76 p. https://agritrop.cirad.fr/596409/

Jagoret P., Michel I., Todem Ngnogué H., Lachenaud P., Snoeck D., Malézieux E., 2017. Structural characteristics determine productivity in complex cocoa agroforestry systems. Agronomy for Sustainable Development 37: 60. https://doi.org/10.1007/s13593-017-0468-0

Jagoret P., Ruf F., Du Castel C., Harmand J.-M., Rafflegeau S., Saj S., Snoeck D., Wibaux T., 2018. L'agroforesterie : des pratiques diversifiées pour la transition agroécologique de la cacaoculture africaine. In La transition agro-écologique des agricultures du Sud. Côte F.-X., Poirier-Magona E. Perret S., Roudier P., Rapidel B., Thirion M.-C. (Eds). Versailles, Editions Quae, Collection Agricultures et défis du monde, pp. 80-104. ISBN 978-27592-2824-9. http://agritrop.cirad.fr/591171/

Saj S., Durot C., Mvondo Sakouma K., Tayo Gamo K., Avana-Tientcheu M.-L., 2017. Contribution of associated trees to long-term species conservation, carbon storage and sustainability: A functional analysis of tree communities in cacao plantations of Central Cameroon. International Journal of Agricultural Sustainability 15: 282-302. https://doi.org/10.1080/14735903.2017.1311764

Saj S., Jagoret P., Etoa L. E., Eteckji Fonkeng E., Tarla J. N., Essobo Nieboukaho J.-D., Mvondo Sakouma K., 2017. Lessons learned from the long-term analysis of cacao yield and stand structure in central Cameroonian agroforestry systems. Agricultural Systems 156: 95-104. https://doi.org/10.1016/j.agsy.2017.06.002

\section{Quelques liens}

Amsterdam Declaration 'Towards Eliminating Deforestation from Agricultural Commodity Chains with European Countries', 2015. Document .pdf archivé (18-01-2017) à https://euandgvc.archiefweb.eu/\#archive Fairtrade. https://www.fairtrade.net/

International Cocoa Organisation (ICCO). https://www.icco.org/

Ministère de la transition écologique et solidaire (France), 2018. Stratégie nationale de lutte contre la déforestation importée 2018-2030 [SNDI]. https://www.ecologique-solidaire.gouv.fr/france-veut-mettre-fin-dici-2030-deforestation-causee-limportation-produits-non-durables-0

Rainforest Alliance. https://www.rainforest-alliance.org/

UTZ, Part of the Rainforest Alliance. https://utz.org/

World Cocoa Foundation (WCF). https://www.worldcocoafoundation.org/ $23^{\text {rd }}$ Conference of the Parties to the United Nations Framework Convention on Climate Change [UNFCCC]. COP23, https://cop23.com.fj/

\section{Directeur de la publication : Michel Eddi, Président directeur général du Cirad Rédacteur.rice.s en chef : Patrick Caron, Umr ART-Dev [Acteurs, ressources et territoires dans le développement] Cécile Fovet-Rabot, délégation à l'information scientifique et technique \\ Étienne Hainzelin, direction générale \\ Mise en pages et illustrations : Laurence Laffont Diffusion : Christiane Mastri, délégation à la communication}

https://revues.cirad.fr/index.php/perspective perspactive ISSN-L 2275-9131 - Email : perspective@cirad.fr

\section{a. M U S E}

MONTPELLER UNVERSTIÉD'EXCELENCE https://muse.edu.umontpellier.fr 